\title{
MUDANÇA DE DECÚBITO EM PACIENTES COM INJÚRIA CEREBRAL GRAVE: CONSTRUÇÃO DE UM GUIA COM ENFERMEIROS INTENSIVISTAS
}

Luciana Machado Magnus¹, Marli Terezinha Stein Backes², Dirce Stein Backes ${ }^{3}$

Objetivo: definir critérios para mudança de decúbito em pacientes com injúria cerebral grave, na perspectiva de enfermeiros de Unidade de Terapia Intensiva, visando a construção de um guia de cuidados. Metodologia: Pesquisa qualitativa exploratóriadescritiva, realizada por meio de Grupo Focal, entre setembro e outubro de 2015, fundamentada no pensamento da complexidade. Foram realizados três encontros com oito enfermeiros de uma Unidade de Terapia Intensiva de referência em neurologia e neurocirurgia. Resultados: Foram construídas duas unidades de análise: "Percepção e avaliação dos enfermeiros frente à mudança de decúbito em pacientes com injúria cerebral grave" e, "Definindo critérios para a elaboração de um guia de mudança de decúbito em pacientes com injúria cerebral grave". Conclusões: Os grupos focais e a análise focal estratégica, aliada ao pensamento da complexidade possibilitaram ampliar a compreensão teórico-prática sobre mudança de decúbito em pacientes neurológicos e neurocirúrgicos, contribuindo para a prática avançada em enfermagem.

Descritores: Injúrias cerebrais, Cuidados de enfermagem, Posicionamento do paciente, Unidades de terapia intensiva.

\section{DECUBITUS CHANGE IN PATIENTS WITH SEVERE BRAIN INJURY FROM THE PERSPECTIVE OF INTENSIVIST NURSES}

Objective: to develop criteria for decubitus change in patients with severe brain injury, in the perspective of nurses of an Intensive Therapy Unit, having the construction of a care guide as goal. Methodology: Qualitative study descriptive-exploratory, conducted through focus groups, between September and October 2015, grounded in the complexity theory thought. There were three meetings with eight nurses of an Intensive Therapy Unit of reference in neurology and neurosurgery. Results: The adopted method allowed the construction of two units of analysis: "Perception and evaluation of nurses regarding the change of decubitus in patients with severe brain injury" and "Defining criteria to the development of a guide for decubitus change in patients with severe brain injury". Conclusions: The focus groups and strategic focal analysis, paired with the complexity theory thought, made it possible to expand the theoretic and practical understanding of decubitus change in neurological patients, contributing to advanced practice nursing.

Keywords: Brain Injuries, Nursing care, Patient positioning, Intensive care units.

\section{EL CAMBIO DE DECÚBITO EN PACIENTES CON GRAVE LESIÓN CEREBRAL DESDE LA PERSPECTIVA DE ENFERMEROS DE LA UNIDAD DE CUIDADOS INTENSIVOS}

Objetivo: Fue establecer criterios para cambio de decúbito en los pacientes con lesión cerebral grave, en la perspectiva de enfermeros de una unidad de cuidados intensivos, para la elaboración de una guía de cuidados. Metodología: Investigación cualitativa exploratoria descriptiva, realizada con la ayuda de grupo focales, entre septiembre y octubre de 2015, que está fundamentada en la idea de la complejidad. Se realizaron tres reuniones con ocho enfermeros de una unidad de cuidados intensivos de referencia en neurología y neurocirugía del sur de Brasil. Resultados: El método adoptado permitió la elaboración de dos unidades de análisis: "La percepción y la evaluación de los enfermeros que se enfrentan al cambio de decúbito de pacientes con lesión cerebral grave" y "La definición de criterios para la elaboración de una guía para el cambio de decúbito en pacientes con lesión cerebral grave". Conclusiones: Los grupos de discusión y la análisis estratégica, junto con la idea de la complejidad, permitieron ampliar el conocimiento teórico y práctico del cambio de decúbito en los pacientes neurológicos, contribuyendo para la práctica avanzada en la enfermería.

Palabras clave: Lesiones cerebrales, Cuidados de enfermeira, Cambio de posición, Unidades de cuidados intensivos 


\section{INTRODUÇÃO}

A injúria cerebral traz consequências devastadoras e, muitas vezes, irreversíveis para o sistema nervoso. Entretanto, os danos causados a esse sistema podem ser evitados e/ou amenizados por enfermeiros intensivistas e/ou especializados em neurologia, com a possibilidade de oferecerem a esses pacientes melhores chances de recuperação르. através da vigilância adequada, aplicação de planos de cuidados e por meio da interação com a equipe ${ }^{2}$.

Sabe-se que a assistência ao paciente neurocrítico é desafiadora e exige muita atenção, especialmente, em relação aos processos intracranianos e à interação entre os transtornos sistêmicos ${ }^{2}$. Sendo assim, é imprescindivel que os enfermeiros de unidades de terapia intensiva neurológicas e neurocirúrgicas tenham conhecimentos de anatomia e fisiologia cerebral, bem como sobre a fisiopatologia da lesão cerebral para assistir o paciente de forma adequada ${ }^{1}$.

Da mesma forma, no processo de cuidado de enfermagem, cabe ao enfermeiro atentar para a segurança do paciente. Para tanto, é fundamental que as condutas profissionais sejam baseadas em protocolos e diretrizes clínicas, pois estes são instrumentos que padronizam e orientam o tratamento e indicam um cuidado respaldado por evidências científicas, a fim de alcançar os melhores resultados ${ }^{3}$.

A mudança de decúbito é um dos cuidados de enfermagem que nos pacientes com quadro neurológico e/ou neurocirúrgico ainda é permeada de dúvidas, especialmente, relacionadas ao momento do início, a periodicidade e posições em que estes pacientes podem permanecer sem que isso traga prejuizos ao seu estado de saúde.

No entanto, as intervenções de enfermagem devem ser baseadas no conhecimento técnico-científico e na avaliação clínica realizada pelo enfermeiro, pois se constituem em ferramentas importantes no planejamento da assistência de forma adequada e intensiva².

Reconhece-se que a mudança de decúbito é um procedimento utilizado para prevenir Lesões por Pressão (LP) e complicações cardiopulmonares, que traz conforto e manutenção do tônus muscular em pacientes com alterações da mobilidade ${ }^{1}$. Entretanto, segundo o autor, a posição do paciente que sofreu injúria cerebral grave é um fator importante a ser considerado pelos enfermeiros, visto que algumas posições poderão interferir no fluxo sanguíneo cerebral, levando ao aumento da pressão intracraniana.

Neste sentido questiona-se: que critérios o enfermeiro deve levar em conta ao prescrever a mudança de decúbito em pacientes com injúria cerebral grave? Com base no exposto, o estudo se justifica pela necessidade de apreender a mudança de decúbito de forma ampla e não fragmentada, de modo a assistir o paciente como um todo e possibilitando-lhe a melhor assistência possivel a partir da elaboração de um guia de cuidados.

\section{OBJETIVO}

Definir critérios para a mudança de decúbito em pacientes com injúria cerebral grave, na perspectiva de enfermeiros de uma Unidade de Terapia Intensiva, visando a construção de um guia de cuidados.

\section{METODOLOGIA}

Pesquisa de abordagem qualitativa, exploratória e descritiva, fundamentada no pensamento da complexidade. Os dados foram coletados por meio da técnica de Grupo Focal (GF) com oito enfermeiros de uma Unidade de Terapia Intensiva (UTI) de um hospital público de referência em neurologia e neurocirurgia do Estado de Santa Catarina, entre o período de setembro e outubro de 2015.

GF caracteriza-se como uma técnica de coleta de dados que promove a comunicação entre os participantes do grupo que trocam experiências, fazem perguntas, dão opiniões, sendo que essa interação em grupo facilita a expressão de ideias e experiências ${ }^{4}$.

Foram realizados três encontros focais com duração de aproximadamente 60 minutos cada, através dos quais buscou-se conhecer a percepção dos enfermeiros sobre a prescrição de mudança de decúbito no paciente com injúria cerebral grave. Além disso, buscou-se discutir sobre as percepções dos enfermeiros com base no pensamento da complexidade.

No primeiro encontro buscou-se conhecer a percepção dos enfermeiros sobre a prescrição de mudança de decúbito ao paciente com injúria cerebral grave. Para isso foi lançado o seguinte questionamento: quais são os critérios que você utiliza para prescrever mudança de decúbito em pacientes internados na UTI acometidos por injúria cerebral grave? Os participantes tiveram um tempo de aproximadamente 15 minutos para escrever ou desenhar suas respostas, caso preferissem e, após, houve a socialização e discussão das percepções.

No segundo encontro se retomou a sintese do primeiro encontro e se buscou discutir sobre as percepções dos enfermeiros com base no pensamento da complexidade. Para tanto, apresentou-se, inicialmente, os principais aspectos do pensamento da complexidade propostos por Edgar Morin.

No terceiro encontro, se retomou a sintese dos encontros anteriores e, a seguir, foram apresentados os resultados da Revisão Integrativa (RI) realizada previamente sobre mudança de decúbito em pacientes com injúria cerebral grave, no sentido de aproveitar estes resultados como subsídios para 
definir conjuntamente os critérios para a elaboração do guia de boas práticas de enfermagem sobre a temática proposta. Ao final da apresentação dos dados da RI foi discutido sobre as evidências obtidas e sobre a prática e experiência de cada profissional presente e, desta forma, foram definidos alguns critérios que irão compor o guia de boas práticas. Todos contribuíram com suas ideias e vivência profissional.

Os critérios para prescrever mudança de decúbito que não foram encontrados na RI foram então definidos de acordo com a experiência de cada profissional presente neste terceiro e último encontro.

A análise dos dados foi realizada com base na Análise Focal Estratégica (AFE), que objetiva a análise ampliada e participativa tanto na coleta quanto na análise dos dados. A AFE propõe a inserção dos participantes como autores e atores no processo de proposição de estratégias, ou seja, os participantes da pesquisa são sujeitos ativos na construção das sinteses e, por final, na construção das unidades de análise ${ }^{5}$

Este estudo foi desenvolvido conforme preconiza a Resolução $n^{\circ}$ 466/12 do Conselho Nacional de Saúde(6). O projeto de pesquisa foi aprovado pelo Comitê de Ética em Pesquisa com Seres Humanos sob o parecernํㅜ 1.163.099/2015 e pela instituição onde foi realizado o estudo. Todos os participantes assinaram do Termo de Consentimento Livre e Esclarecido.

\section{RESULTADOS}

Participaram do estudo oito enfermeiros intensivistas. Destes, dois atuavam na referida UTI há mais de 20 anos, um há nove anos e um há sete anos e, os demais, entre um a quatro anos. Dos oito participantes, sete possuiam pósgraduação a nivel de especialização, três eram mestres em enfermagem e um estava cursando mestrado.

A realização dos três encontros por meio do GF possibilitou a troca de experiências e reflexões sobre as melhores práticas no que se refere à mudança de decúbito no paciente que sofreu injúria cerebral grave. A partir disso, foram elaboradas duas unidades de análise que serão apresentadas a seguir.

\section{Percepção e avaliação dos enfermeiros frente à mudança de decúbito em pacientes com injúria cerebral grave}

Alguns enfermeiros evidenciaram os benefícios da mudança de decúbito para prevenir LP. Outras percepções foram associadas à preocupação dos enfermeiros com as condições clínicas do paciente e a importância de se avaliar o paciente antes da realização da mudança de decúbito. Sendo assim, a maioria dos enfermeiros referiu que antes de realizar a prescrição do procedimento avalia o bem estar do paciente, incluindo sua condição clínica, sem desmerecer a importância da mudança de decúbito.

Concluiu-se que a mudança de decúbito apresenta riscos e benefícios. Quando o enfermeiro prescreve mudança de decúbito está pensando nos benefícios, como prevenção de LP e complicações pulmonares, porém, ao mesmo tempo, o enfermeiro deve avaliar se a mudança de decúbito não irá prejudicar a condição clínica do paciente com injúria cerebral grave.

\section{Definindo critérios para a elaboração do guia de mudança de decúbito em pacientes com injúria cerebral grave}

Para a definição dos critérios de mudança de decúbito para a construção do guia a ser utilizado na unidade de estudo, foram considerados os dados encontrados na revisão integrativa e a vivência teórico-prática dos participantes. Esses critérios serão apresentados na sequência, por meio de Quadros

O Quadro 1 apresenta os critérios definidos para a mudança de decúbito para paciente com monitorização de Pressão Intracraniana (PIC), considerando os parâmetros a serem avaliados, a periodicidade da mudança e a posição a ser adotada, identificando a fonte, enfermeiro (peritos) ou revisão integrativa, e o nivel de evidência conforme Polit e Beck ${ }^{7}$.

Quadro 1: Critérios definidos para a mudança de decúbito para paciente com monitorização de PIC, Florianópolis, 2015

\begin{tabular}{|c|c|c|c|c|c|}
\hline $\begin{array}{c}\text { Mudança de } \\
\text { decúbito }\end{array}$ & $\begin{array}{c}\text { Parämetros a } \\
\text { serem avaliados }\end{array}$ & Periodicidade & Decúbitos & Fonte & $\begin{array}{c}\text { Nivel de evidência } \\
\text { conforme Pelite } \\
\text { Beck (2011) }\end{array}$ \\
\hline $\begin{array}{|lr|}\begin{array}{l}\text { Iniciar } \\
\text { mudança }\end{array} & \text { de } \\
\text { decúbito } & 6 \\
\text { horas após } & \text { a } \\
\text { chegada } & \text { do } \\
\text { paciente } & \text { do } \\
\text { Centro } & \\
\text { Cirúrgico na } \\
\text { UTI }\end{array}$ & 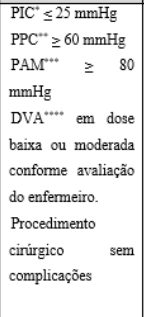 & $\begin{array}{l}\text { A cada duas } \\
\text { horas }\end{array}$ & $\begin{array}{l}\text { DLD (decúbito lateral } \\
\text { direito) } \\
\text { DLE (decúbito lateral } \\
\text { esquerdo) } \\
\text { DD (decúbito dorsal) } \\
\text { OBS: manter a linha } \\
\text { média da cabeça com a } \\
\text { cabeceira do leito } \\
\text { elevada a } 30^{\circ} \\
\text { independente do } \\
\text { decúbito }\end{array}$ & \begin{tabular}{l} 
Estudo da RI \\
(Kosere e \\
Hatipeglu. \\
2012) \\
\multicolumn{1}{c}{ e } \\
\\
de acordo com \\
os Enfermeiros \\
Intensivistas
\end{tabular} & $\begin{array}{l}\text { VII - critérios } \\
\text { clinicos e experiência } \\
\text { de Especialistas }\end{array}$ \\
\hline $\begin{array}{|lr|}\text { Não iniciar } \\
\text { mudança } & \text { de } \\
\text { decúbito } & \text { se } \\
\text { instabilidade } & \\
\text { hemodinámica } \\
\text { e HIC por } \\
\text { periodo } \\
\text { prolongado }\end{array}$ & \begin{tabular}{|l|}
$\mathrm{PIC} \geq 25 \mathrm{mmHg}$ \\
$\mathrm{PPC} \leq 60 \mathrm{mmHg}$ \\
$\mathrm{PAM} \leq 80 \mathrm{mmHg}$ \\
Hemodinâmica \\
instável, dependente \\
de DVA em dose \\
elevada conforme \\
avaliação do \\
enfermeiro. \\
Complicação \\
cinúrgica *...
\end{tabular} & \begin{tabular}{|l|} 
Realizar \\
massagem de \\
conforto a \\
cada 4 horas
\end{tabular} & $\begin{array}{l}\text { Manter o paciente em } \\
\text { decúbito dorsal com } \\
\text { leve elevação dos } \\
\text { joelhos }\end{array}$ & $\begin{array}{l}\text { Estudo da RI } \\
\text { (Ledwith et al., } \\
\text { 2010) } \\
\quad \text { e } \\
\\
\text { de acordo com } \\
\text { os Enfermeiros } \\
\text { Intensivistas }\end{array}$ & $\begin{array}{l}\text { IIA - pelo menos um } \\
\text { ensaio clínico } \\
\text { controlado } \\
\text { Randomizado } \\
\text { VII- critérios clínicos } \\
\text { e experiência de } \\
\text { Especialistas }\end{array}$ \\
\hline
\end{tabular}

Fonte: Grupo focal realizado com enfermeiros.

${ }^{*} \mathrm{PIC}=$ Pressão Intracraniana

${ }^{* *} \mathrm{PPC}=$ Pressão de perfusão cerebral

*** PAM $=$ Pressão Arterial Média

**** DVA = Droga Vasoativa

${ }^{* * * *}$ Avaliar o tempo de cirurgia, sangramento durante a cirurgia, caracteristica da lesão e prognóstico. 
O Quadro 2 a seguir destaca os critérios definidos para a mudança de decúbito em pacientes sem monitorização de PIC, considerando, da mesma forma, os parâmetros a serem avaliados, a periodicidade da mudança e a posição a ser adotada, identificando a fonte, enfermeiro (peritos) ou revisão integrativa, e o nivel de evidência conforme Polit e Beck

Quadro 2 - Critérios definidos para a mudança de decúbito em paciente sem monitorização de Pressão Intracraniana (PIC), Florianópolis, 2015

\begin{tabular}{|c|c|c|c|c|c|}
\hline $\begin{array}{l}\text { Quando iniciar } \\
\text { mudança de } \\
\text { decúbito }\end{array}$ & $\begin{array}{l}\text { Parâmetros a serem } \\
\text { avaliados }\end{array}$ & $\begin{array}{l}\text { Periodicidad } \\
e\end{array}$ & Decúbitos & Fonte & $\begin{array}{ll}\begin{array}{l}\text { Nivel } \\
\text { evidência }\end{array} & \text { de } \\
& \end{array}$ \\
\hline $\begin{array}{l}\text { Pacientes sem } \\
\text { evidências de } \\
\text { HIC iniciar } \\
\text { imediatamente } \\
\text {. }\end{array}$ & $\begin{array}{l}\text { Avaliar a clínica da } \\
\text { HIC" (bradcardia } \\
\text { alteração de pupilas e } \\
\text { elevação da pressão } \\
\text { arterial). } \\
\text { TC"* de crânio } \\
\text { Hemodinâmica } \\
\text { Avaliação } \\
\text { neurológica } \\
\text { (GLASGOW*.. e } \\
\text { pupilas). } \\
\text { Sedação profunda ou } \\
\text { superficial }\end{array}$ & 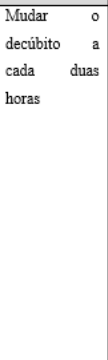 & $\begin{array}{l}\text { DLD (decúbito } \\
\text { lateral direito) } \\
\text { DLE (decúbito lateral } \\
\text { esquerdo) } \\
\text { DD (decúbito dorsal) } \\
\text { OBS: manter a linha } \\
\text { média da cabeça com } \\
\text { a cabeceira do leito } \\
\text { elevada a } 30^{\circ} \\
\text { independente do } \\
\text { decúbito }\end{array}$ & 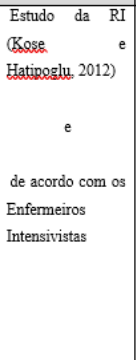 & $\begin{array}{l}\text { IIA - ensaio } \\
\text { clínico } \\
\text { Controlado } \\
\text { randomizado } \\
\\
\text { VII - critérios } \\
\text { clínicos e } \\
\text { experiência de } \\
\text { Especialistas }\end{array}$ \\
\hline $\begin{array}{l}\text { Pacientes com } \\
\text { HIC grave não } \\
\text { iniciar. }\end{array}$ & $\begin{array}{l}\text { Avaliar a clínica da } \\
\text { HIC (bradicardia, } \\
\text { alteração de pupilas e } \\
\text { elevação da pressão } \\
\text { arterial). } \\
\text { TC de crânio } \\
\text { Hemodinâmica } \\
\text { Avaliação } \\
\text { neurológica } \\
\text { (GLASGOW } \\
\text { pupilas). } \\
\text { Sedação profunda ou } \\
\text { superficial }\end{array}$ & $\begin{array}{l}\text { Realizar } \\
\text { massagem de } \\
\text { conforto a } \\
\text { cada } 4 \text { horas }\end{array}$ & $\begin{array}{l}\text { Manter o paciente em } \\
\text { decúbito dorsal com } \\
\text { leve elevação dos } \\
\text { joelhos }\end{array}$ & $\begin{array}{l}\text { Estudo da RI } \\
\text { (Ledxith et al, } \\
\text { 2010) } \\
\qquad \text { e } \\
\text { de acordo com os } \\
\text { Enfermeiros } \\
\text { Intensivistas }\end{array}$ & $\begin{array}{l}\text { IIA - pelo menos, } \\
\text { um ensaio clínico } \\
\text { controlado } \\
\text { Randomizado } \\
\\
\text { VII- critérios } \\
\text { clínicos e } \\
\text { experiência de } \\
\text { Especialistas }\end{array}$ \\
\hline
\end{tabular}

Fonte: Grupo focal realizado com enfermeiros.

${ }^{*} \mathrm{HIC}=$ hipertensão Intracraniana

${ }^{\star *} \mathrm{TC}=$ Tomografia Computadorizada

***GLASGOW = Escala de Coma de Glasgow. É uma escala neurológica que parece constituir-se num método confiável e objetivo de registrar o nível de consciência de uma pessoa, para avaliação inicial e contínua após um traumatismo craniano.

O Quadro 3 aponta os critérios definidos para a mudança de decúbito em pacientes com craniectomia descompressiva, considerando igualmente os parâmetros a serem avaliados, a periodicidade da mudança e a posição a ser adotada, identificando a fonte e o nível de evidência, conforme Polit e Beck $^{7}$ e observações.
Quadro 3 - Critérios definidos para a mudança de decúbito em pacientes com craniectomia descompressiva, Florianópolis,

2015

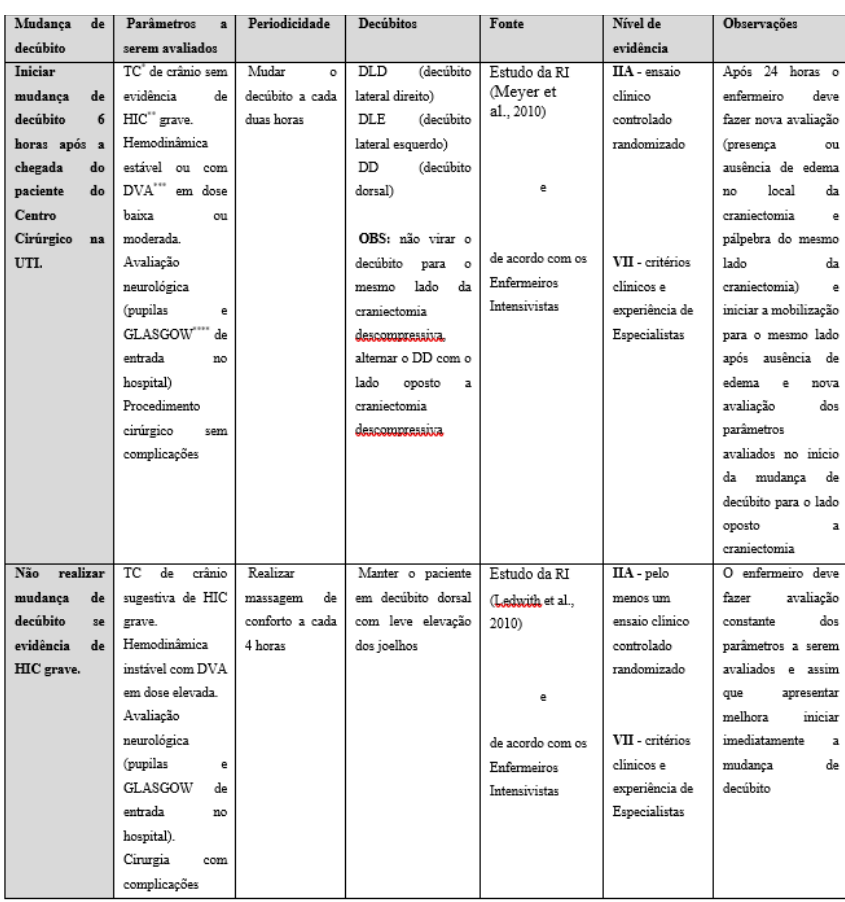

Fonte: Grupo focal realizado com enfermeiros.

* $\mathrm{TC}=$ Tomografia Computadorizada

** HIC = hipertensão intracraniana

${ }^{* * *}$ DVA $=$ Droga Vasoativa

****GLASGOW = Escala de Coma de Glasgow. É uma escala neurológica que parece constituir-se num método confiável e objetivo de registrar o níve de consciência de uma pessoa, para avaliação inicial e contínua após um traumatismo craniano.

Os critérios amplamente discutidos e aqui apresentados poderão servir de guia de orientação e abertura para novas possibilidades teórico-práticas. O enfermeiro deverá estar sempre reavaliando as condições clínicas do paciente para gerenciar da melhor forma possivel a mudança de decúbito, evitando agravos causados pela mudança de decúbito tardia e/ou inadequada, visando a segurança ao paciente.

\section{DISCUSSÃO}

A mudança de decúbito é um procedimento que requer do enfermeiro avaliação constante e individualizada, a partir de um raciocínio clínico que não deve ser simplificador. 
Embora esse procedimento tenha muitos benefícios para a pele e função pulmonar do paciente, em cuidados intensivos a condição clínica do paciente com injúria cerebral é um fator a ser avaliado pelo enfermeiro antes de prescrever a mudança de decúbito.

Desta maneira, fica evidente que a mudança de decúbito trata-se de um procedimento complexo na UTI e que não pode ser realizada de forma mecanizada em todos os pacientes da mesma forma, ou seja, de duas em duas horas (ordem), sem considerar as individualidades (partes) e o contexto de saúde de cada paciente.

O enfermeiro deve avaliar as condições clínicas para que o efeito deste procedimento não cause instabilidade e piora no paciente. No ambiente hospitalar e, em especial, nas unidades de terapia intensiva, na maioria das vezes, são estabelecidas rotinas rígidas que tem horário para o início e previsão para o término. Porém, em algumas situações, o enfermeiro, ao avaliar a condição do paciente (contexto) pode observar que é necessário evitar a realização da mudança de decúbito no momento rotineiro devido a instabilidade do paciente, visto que o procedimento poderá contribuir para hipertensão intracraniana grave (desordem).

Nesta direção, Morin destaca que a complexidade nos torna prudentes, atentos, e nos mostra que não devemos nos fechar na crença de que o que acontece hoje vai continuar indefinidamente ${ }^{8}$, ou seja, um paciente com injúria cerebral pode apresentar instabilidades intercalados com melhora clínica. Por esse motivo, precisamos superar os determinismos prévios e nos mostrar abertos ao pensamento complexo que nos ajuda a entender e contextualizar melhor cada situação e nos leva a agir de forma mais coerente e eficaz.

Morin complementa ainda que "o pensamento complexo não recusa a clareza, a ordem, o determinismo, e sim, os considera insuficientes uma vez que não se pode programar a descoberta, o conhecimento e a ação". Desse modo, o pensamento complexo traz um lembrete: "não esqueça que a realidade é mutante, que o novo pode surgir e vai surgir"(8:83)

Estudo de revisão sistemática realizado no período 2003 a 2015 destacou a mudança de decúbito como uma "estratégia

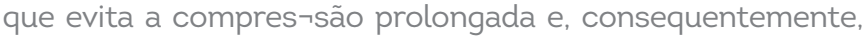
a redução da irrigação sanguínea local"(9:28). Os autores afirmam que a mudança de decúbito deve ser realizada, pelo menos, a cada duas horas, para evitar LP, desde que não haja contraindicações.

Os pacientes que apresentam quadro neurológico e/ou neurocirúrgico, ou que possuem o seu nível de consciência diminuído por permanecerem mais tempo acamados, com sua mobilidade reduzida e a maior parte do tempo com a cabeceira da cama levantada a 30으, e em decúbito dorsal, apresentam predisposição aumentada para o desenvolvimento de LP'
Estudo transversal realizado com 1.762 pacientes durante o período de dezembro de 2012 a abril de 2013 obteve na UTI uma incidência maior de LP (44,83\%) do que nos demais setores de internação(10). No entanto, os autores destacam que nas unidades de terapia intensiva houve o predomínio de patologias cerebrovasculares (26,6\%), seguida de doenças respiratórias $(22,2 \%)$, que são frequentes em pacientes críticos, o que contribui para a instabilidade hemodinâmica e também limita a mobilidade, levando esses pacientes a permanecerem em repouso prolongando durante o tempo de internação.

No entanto, os pacientes com quadro neurológico e/ ou neurocirúrgico também podem e devem ser mudados de decúbito, sendo que para isso é preciso tomar alguns cuidados, considerando a prescrição realizada pelo enfermeiro. Existem indicações de posições para esses pacientes, mesmo que eles sejam mantidos em decúbito dorsal e com a cabeceira elevada a 30으, como o decúbito lateral direito e esquerdo, mantendo a cabeça alinhada ao corpo, e elevar os joelhos.

Um estudo clínico experimental testou os efeitos da posição da cabeça e do pescoço no fluxo sanguíneo cerebral por Doppler Transcraniano em 38 pacientes $^{11}$. Os dados sugerem que tanto a posição lateral direita quanto a esquerda são seguras e recomendadas quando não houver contraindicação.

A mudança de decúbito não é a única maneira de realizar a mobilização em pacientes acamados na UTI. Também existem exercícios passivos, ativo-assistidos e ativos, além de outros meios complementares, como a eletroestimulação neuromuscular e a prancha ortostática que também são consideradas eficazes e geram beneficios aos pacientes ${ }^{12}$.

As equipes de enfermagem atuantes nas unidades de terapia intensiva que mantém os pacientes a maior parte do tempo na posição dorsal, podem não estar atualizadas sobre esse dado ou estão cometendo iatrogenia?. Os autores complementam que essa situação acaba gerando mais prejuizos ao paciente que acaba desenvolvendo LP durante a sua internação, e também aumenta os custos com materiais para o tratamento deste agravo.

Estudo realizado evidenciou que "a mobilização precoce em pacientes críticos promove benefícios físicos, psicológicos, evita riscos gerados pela hospitalização prolongada, acelerando a recuperação e reduzindo a incidência sobre complicações pulmonares e musculoesqueléticas"(12:180). Os autores apontam como estratégias para a mobilização precoce nos pacientes a mudança de decúbito, a mobilização passiva, a deambulação, o ortostatismo e a eletroestimulação neuromuscular

Nessa direção, estes mesmos autores complementam que a internação prolongada em UTI traz prejuízos aos pacientes, 
pois "gera problemas como imobilidade, descondicionamento físico e fraqueza muscular"(12:177). Os autores defendem que a mudança de decúbito deve ser realizada precocemente por trazer benefícios aos pacientes, mas também referem que é fundamental saber quais as repercussões e controvérsias relacionadas com a mobilização precoce em pacientes internados na UTI: "a mobilização precoce é a base para a recuperação funcional, pois otimiza o transporte de oxigênio, mantém a força muscular, reduz efeitos do imobilismo, a permanência na UTI e promove melhora na qualidade de vida após a alta hospitalar. Esse procedimento será seguro e viável quando as barreiras encontradas como sedação, analgesia e bloqueio neuromuscular forem cessadas ou diminuídas, visto que contribuem para o agravamento dos resultados clínicos"(12:177).

Os dados de um estudo prospectivo, observacional quaseexperimental propõe que os pacientes sejam cuidadosamente monitorizados quando estiverem na posição lateral direita ou esquerda. Os autores acreditam que os dispositivos para monitorização da PIC podem ajudar a determinar a melhor posição para cada paciente ${ }^{13}$, de forma a trazer segurança ao enfermeiro que prescreve esse cuidado, uma vez em que, se ocorrer hipertensão intracraniana (HIC) em determinado decúbito, por período de curta duração, há indicação de retornar ao decúbito anterior sem trazer malefícios ao paciente.

Os dados dessa mesma pesquisa revelam que pode não haver posição ideal para o paciente com injúria cerebral que diminua a PIC, aumente a Pressão de Perfusão Cerebral (PPC) ou o oxigênio no cérebro concomitantemente. Nessa direção, essa pesquisa indica a utilização de cateteres de monitorização de PIC para ajudar a determinar a melhor posição para cada paciente individualmente e, assim, ajudar a diminuir a lesão secundária. Porém, na ausência de cateteres de monitorização de PIC os autores acreditam que a melhor posição para diminuir a PIC e aumentar a PPC seja o decúbito dorsal, com a elevação da cabeceira da cama a 30ㅇe com leve elevação dos joelhos.

De qualquer maneira, é preciso ter em mente os fatores limitantes e também as barreiras impostas para que a mobilização precoce dos pacientes seja aplicada com segurança ${ }^{12}$. Estudo realizado em uma UTI de uma instituição privada de Campos dos Goytacazes no ano de 2014 apontou que há deficiências nos cuidados de enfermagem realizados em pacientes neurocríticos, como no caso dos portadores de acidente vascular encefálico hemorrágico(2). Para garantir a segurança na assistência, os autores recomendam a implementação de um protocolo uma vez em que o seu uso diminui margens de erros a partir da padronização do cuidado.

Da mesma forma, estudo realizado em um hospital universitário no Sul do Brasil, no ano de 2014, com 22 enfermeiros lotados na UTI Adulto, Unidade Cardíaca Intensiva e Clínica Médica reforça a importância da utilização de protocolos na assistência e aponta que no gerenciamento do cuidado de enfermagem ao paciente com LP, o uso de protocolo como instrumento gerencial agrega qualidade à assistência de enfermagem ${ }^{14}$.

Em suma, tornam-se necessários mais estudos para se conhecer melhor as indicações, barreiras, contraindicações da mobilização precoce, assim como saber quais os riscos e os efeitos causados por esta mobilização nos pacientes críticos internados nas UTI, bem como a elaboração de protocolos para orientar a realização do procedimento, e treinamentos com a equipe ${ }^{12}$.

Como limitação pode-se citar os poucos artigos que abordam a mudança de decúbito em pacientes com injúria cerebral, o que implicou em pouca produção científica para subsidiar os critérios a serem utilizados para prescrever mudança de decúbito a esses pacientes.

\section{CONCLUSÃO}

A reflexão sobre a complexidade que envolve a mudança de decúbito em pacientes neurológicos e/ou neurocirúrgicos permitiu a definição de alguns critérios baseados na literatura e experiência dos enfermeiros intensivistas que servirão de subsídios para a construção do Guia de cuidados para mudança de decúbito no paciente que sofreu injúria cerebral grave internado na UTI, visando contribuir para a qualificação do cuidado de enfermagem.

O método adotado aliado ao pensamento da complexidade possibilitou ampliar a compreensão teórico-prática sobre a mudança de decúbito em pacientes neurológicos e transcender a linearidade do fazer tradicional fragmentado e reducionista e, dessa forma, contribui para a prática avançada em enfermagem. 


\section{REFERÊNCIAS}

1. Cintra EA, Littlejohns S, Malta MA, Diccini S. O Papel da Enfermagem no Neurointensivismo. In: Terzi R, Falcão A, Videtta W. Cuidados neurointensivos. Clínicas de Medicina Intensiva Brasileira. Ano 18. v.20. São Paulo: Atheneu, 2013

2. Barcelos DG, Santos CM, Manhães LSP, Azevedo AS. Atuação do enfermeiro em pacientes vitimas do acidente vascular encefálico hemorrágico na unidade de terapia intensiva. Persp. Online: biol. \& saúde. 2016 [cited 2018 Feb 14]; 22(6):41-53. Available from: http://www.seer. perspectivasonline.com.br/index.php/biologicas_e_saude/article/ view/1097.

3. Marques CRG, Ferrari YAC, Oliveira CGS. Atuação do enfermeiro no acidente vascular encefálico: uma revisão integrativa. Ciências Biológicas e da Saúde. 2017 [cited 2018 Feb 14]:4(2):127-42. Available from: https://periodicos.set.edu.br/index.php/cadernobiologicas/article/ view/4599.

4. Kitzinger J. Grupos Focais. In: Mays N, Pope C. Pesquisa qualitativa na atenção a saúde. 3. ed. Porto Alegre: Artmed, 2009.

5. Backes DS, Colomé JS, Erdmann RH, Lunardi VL. Grupo focal como técnica de coleta e análise de dados em pesquisas qualitativas. Mundo Saúde. 2011 [cited 2017 Jun 20];35(4):438-42. Available from: http:// bvsms.saude.gov.br/bvs/artigos/grupo_focal_como_tecnica_coleta_ analise_dados_pesquisa_qualitativa.pdf.

6. Brasil. Conselho Nacional de Saúde. Ministério da Saúde. Resolução CNS nㅇ 466/2012. Brasil, 2012. Disponivel em: http://conselho.saude. gov.br/web_comissoes/conep/index.html. Acesso em: 16 outubro 2013.

7. Polit DF, Beck CT. Fundamentos de pesquisa em enfermagem: avaliação de evidências para a prática da enfermagem. 7ạ ed. Porto Alegre: Artmed, 2011
8. Morin E. Introdução ao pensamento complexo. $4^{a}$ ed. Porto Alegre: Sulina, 2011

9. França JRG, Sousa BVN, De Jesus VS. Cuidados de Enfermagem na Prevenção de Lesões por Pressão em Unidades de Terapia Intensiva: uma Revisão Sistemática. Revista Brasileira de Saúde Funcional. 2016 [cited 2017 Feb 23]:1(11):16-31. Available from: www.seer-adventista. com.br/ojs/index.php/RBSF/article/download/709/619.

10. Lainseck FGT, Rodrigues LHN, Ramos TM, Silva KCC, Muniz CF, Barcellos VM. Incidência de úlceras de pressão no hospital regional de Gurupi-TO. Rev. Cereus. 2016 [cited 2017 Feb 23];8(1):182-99. Available from: HTTP://ojs.unirg.edu.br/index.php/l/index.

11. Kose G, Hatipoglu S. Effect of head and body positioning on cerebral blood flow velocity in patients who underwent cranial surgery. J Clin Nurs. 2012 [cited 2017 Feb 23];21(13-14):1859-67. Available from: https:// www.ncbi.nlm.nih.gov/pubmed/22672454.

12. Sarti TC, Vecina MVA, Ferreira PSN. Mobilização precoce em pacientes críticos. J Health Sci Inst. 2016 [cited 2017 Feb 19];34(3):177-82. Available from: https://www.unip.br/comunicacao/publicacoes/ics/edicoes/2016/03_jul-set/V34_n3_2016_pl77al82.pdf.

13. Ledwith MB, Bloom S, Maloney-Wilensky E, Coyle B, Polomano RC Le Roux PD. Effect of body position on cerebral oxygenation and physiologic parameters in patients with acute neurological conditions. $\mathrm{J} \mathrm{Neu-}$ rosci Nurs. 2010 [cited 2017 Feb 23];42(5):280-7. Available from: https:// www.ncbi.nlm.nih.gov/pubmed/20968224.

14. Soares RSA, Lima SBS, Camponogara S, Eberhardt TD, Fonseca GGP Kessler M. Significado do protocolo de úlcera por pressão: qualificando a gerência do cuidado do enfermeiro. Enferm Foco [Internet]. 2017 [cited 2017 Dec 20];8(3):19-24. Available from: http://revista.portalcofen. gov.br/index.php/enfermagem/article/view/859/393. 